\title{
DEVELOPMENT OF AN OPTICAL DEVICE TO INVESTIGATE CHLOROPHYLL CONTENT OF TOMATO LEAVES
}

\author{
Di Cui, Minzan $\mathrm{Li}^{*}$, Xiuhua Li \\ Key Laboratory of MOE on Modern Precision Agriculture System Integration \\ Research, China Agricultural University, Beijing 100083, China \\ *Corresponding Author-Tel: +86-10-62737924,Email: limz@cau.edu.cn
}

\begin{abstract}
Chlorophyll content is an important indication for evaluating crop growth status and predicting crop yield. The NDVI (Normalized Difference Vegetation Index) is commonly used as an indicator in practical crop healthy monitoring. Hence, a spectroscopy-based device for indirectly measuring crop growth conditions in terms of NDVI is developed. This device consists of four channels: two are designed to measure the intensity of the sunlight and the other two are used to measure the reflected light from the crop canopy at the same time. An electronic control unit was designed to control the sensing and data recording processes, as well as to calculate the NDVI based on the sensed data. The measurable two wavelengths are $610 \mathrm{~nm}$ and $1220 \mathrm{~nm}$. A series validation tests, comparing the measurement result against spectroradiometer readings, are conducted to evaluate the performance of the device. Leaf samples are collected to measure chlorophyll contents in laboratory. The correlation coefficient between the NDVI readings from the developed device and the chlorophyll content data measured by the UV-VIS Spectrophotometer reaches 0.81 , which shows that the device can be used in practical crop management.
\end{abstract}

Keywords: NDVI, crop growth condition, chlorophyll content, sensor

\section{INTRODUCTION}

Crop chlorophyll content is an important index used to evaluate the growth status and to predict the yield and the quality for many crops.

Please use the following format when citing this chapter:

Cui, D., Li, M. and Li, X., 2009, in IFIP International Federation for Information Processing, Volume 295, Computer and Computing Technologies in Agriculture II, Volume 3, eds. D. Li, Z. Chunjiang, (Boston: Springer), pp. 2111-2118. 
Previous studies revealed that chlorophyll had a great effect on the spectral reflectance of crop leaves (Cartelat et al., 2005). When the nitrogen content is deficient in crop plants, the chlorophyll level in those plants will go down. It will cause a reduction in leaf spectral reflectance at NIR band and an increase at visible band ( $\mathrm{Li}$ et al., 2006). Such a phenomenon makes it possible to estimate crop nitrogen stress in terms of the measurable leave spectral reflectance of crop canopy.

Since 1970s, scientists have conducted many basic researches to evaluate crop growth condition by using spectral reflectance of leaf or canopy. And researches on developing ground-based sensing systems for detecting plants nitrogen content have been reported. Stone et al. (1996) reported the development of optical sensors for detection of nitrogen availability in winter wheat plants. They reported that that device could provide a close correlation between the plant nitrogen contents and a plant nitrogen spectral index (PNSI) calculated as $\mid(N I R+$ red $) /(N I R-$ red $) \mid$. Kim et al. (2001) investigated applicability of using a multispectral camera as a ground-based sensor for assessing corn plant nitrogen stress, also found it could provide a reasonable correlation between NDVI and leaf chlorophyll content. Sui et al. (2005) developed a device for detecting nitrogen status in cotton by measuring the spectral reflectance of cotton canopy in four wavebands (blue, green, red, and NIR) with modulated illumination to the canopy under the Sunlight. Min et al. (2006) investigated the use of a visible and near infrared spectroscopy to estimate nitrogen concentrations of Chinese cabbage. Campbell et al. (2007) investigated the use of reflectance (R) and fluorescence $(F)$ information to monitor the vegetation stress responses. Heege and Thiessen (2002) developed an on-the-go control system for sitespecific nitrogen top dressing in terms of measured crop reflectance. Noh et al. (2005) developed an equipment-mount multispectral image sensor to detect corn plant nitrogen deficiency in real-time for supporting sensor-based variable-rate nitrogen side dressing.

On the other hand, it is necessary to develop a portable detector for small scale farm in China. In order to create a handy device for in-field use, the overall objective of this study was to design a spectra-based hand-hold device to measure spectral reflectance of crop canopy and the sunlight at Red and NIR wavebands, and then calculate NDVI to indirectly detect the chlorophyll content in crop plants. 


\section{METHODOLOGY AND PROCEDURE}

\subsection{Theoretical background for design}

Several vegetation indices have been developed for revealing crop growing condition information. A widely used index is the NDVI (Normalized Difference Vegetation Index). NDVI is calculated as a ratio differences between measured canopy reflectance in the red and near infrared bands, respectively. These two spectral bands are chosen because they are mostly affected by the absorption of chlorophyll in leafy green vegetation. The equation calculating NDVI is as follow (Tucker,1979).

$$
\mathrm{NDVI}=\frac{R_{\mathrm{IR}}-R_{\mathrm{R}}}{R_{\mathrm{IR}}+R_{\mathrm{R}}}
$$

where, $R_{\mathrm{R}}$ and $R_{\mathrm{IR}}$ are canopy reflectance data in the red and near infrared bands, respectively.

\subsection{Sensing unit design}

In order to obtain $R_{\mathrm{R}}$ and $R_{\mathrm{IR}}$ data shown in Equation (1), an optical sensing device was designed, It consisted of four channels: two were designed to measure the intensity of the sunlight and the other two measured the reflected light from the crop canopy at the same time. An electronic control unit was designed to control the sensing and data recording processes, as well as to calculate the NDVI based on the sensed data. Figure 1 illustrates the system schematics of the designed device.

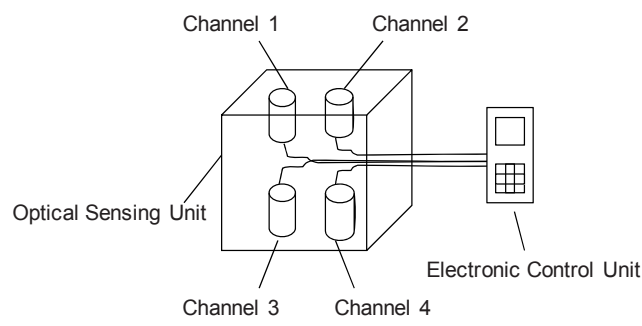

Figure1. The system schematics of designed device

\subsubsection{Design of optical sensing unit}

As introduced above, the designed optical sensing device used four channels for acquiring light reflectance data (Figure 1). Those sensing 
channels were designed identically, each consisting of an optical window for collecting the light, a filter for transmitting only the light of specific wavelength, a lens for making the light get together, and a transducer for converting the optical signal into electrical signal. The structure of each channel is shown in Figure 2.

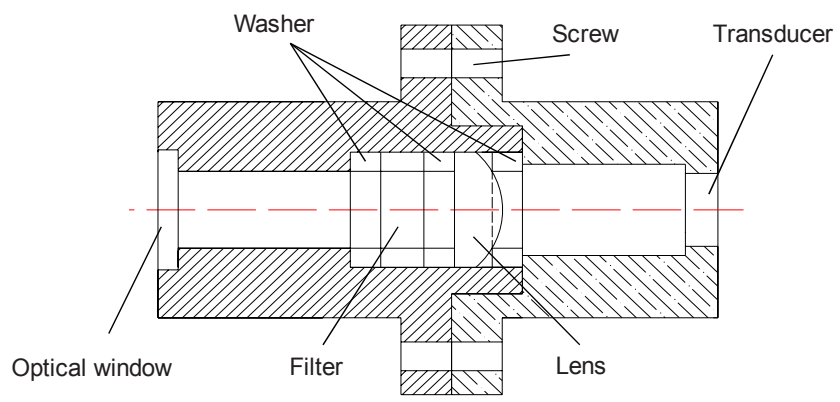

Figure2. The structure of each channel

The optical windows of two channels were configured upwards to measure the intensity of the sunlight, and the other two windows pointed downwards to sense the intensity of the reflected light from plant canopy. Each pair of the opposite arranged channels equipped the same filters and transducers. Since the $610 \mathrm{~nm}$-centered red band and 1220nm-centered NIR band were reported being sensitive to chlorophyll content variation of crop plants (Xue et al., 2004), these two bands were chosen for constructing the optical sensing unit. The type of the filters in channel 1 and 3 (NIR band) was $1220 \pm 6 \mathrm{~nm}$ center wavelength of $20 \mathrm{~mm}$ diameter, and the type of transducers was InGaAs photodiode with a light active area of $1.5 \times 1.5 \mathrm{~mm}^{2}$ and the spectral response range of 1000-1650 nm. Similarly, in channel 2 and 4 (red band) the type of filters was $610 \pm 6 \mathrm{~nm}$ center wavelength of 20 $\mathrm{mm}$ diameter, and the type of transducers was Silicon photocell transducers with a light active area of $5 \times 5 \mathrm{~mm}^{2}$ and a spectral response range of 400 $1100 \mathrm{~nm}$. To reduce the influence of solar elevation angle on transducer outputs, a milky diffuse glass was used as the optical window for two upview channels.

\subsubsection{Design of electronic control unit}

The electronic control unit was designed to control the sensing process, to convert the light induced electric currents in optical sensing elements to voltage signals, and to calculate crop NDVI in terms of the recorded voltage signals. Figure 3 shows the system schematics of the unit. 
The converter changed the electric current into the voltage and the amplifier changed the amplitude of voltage into $0-5 \mathrm{~V}$. The $\mathrm{A} / \mathrm{D}$ transducer converted this analog signal into the digital one, so that the digital signal could be measured by the microprocessor. The microprocessor measured the voltages of the four channels, calculated the NDVI using these four values and sent the results to the LCD.

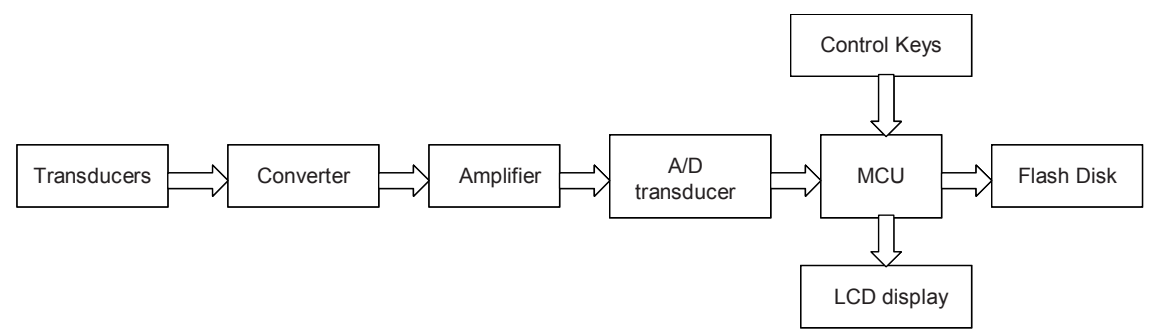

Figure 3. The system schematics of the electronic control unit

\section{TESTS AND RESULTS ANALYSIS}

\subsection{Repeatability analysis}

Before using the developed device in detecting crop plant reflectance to indirectly measure crop nitrogen stress, a performance test was conducted to check the repeatability of the device in measuring the known reflectance. According to the design requirement, the standard deviations $(\sigma)$ of the measured data should be less than 0.1 . In this performance test, device data were collected in a 20 minutes interval between 10:00 AM and 15:40 PM. Each measurement was repeated three times and the average was taken as the measured datum. A white panel of $100 \%$ reflectance and a grey panel of $35 \%$ reflectance were put on the ground out of the shadow as the known reference. The device was perpendicularly positioned to the panel at $10 \mathrm{~cm}$ above the panels.

As shown in Figure 4, the measured reflectancedata at the NIR and red bands are closely distributed within a small range around 1.0 between 10:00 AM and 14:40 PM, but those data experienced a dramatic increase after 5:00 PM. Through visual observation, the intensity of sunlight was noticeably decreased after 15:00 PM. In addition, since a milky diffuse glass was used as the optical window for two up-view channels, when intensity of sunlight decreased, the light entered the up-view channels would much more 
decreased than that in down-view channels. This result revealed that without any additional compensation the developed device could provide a reasonable repeatability when the sunlight intensity is above a certain threshold value.

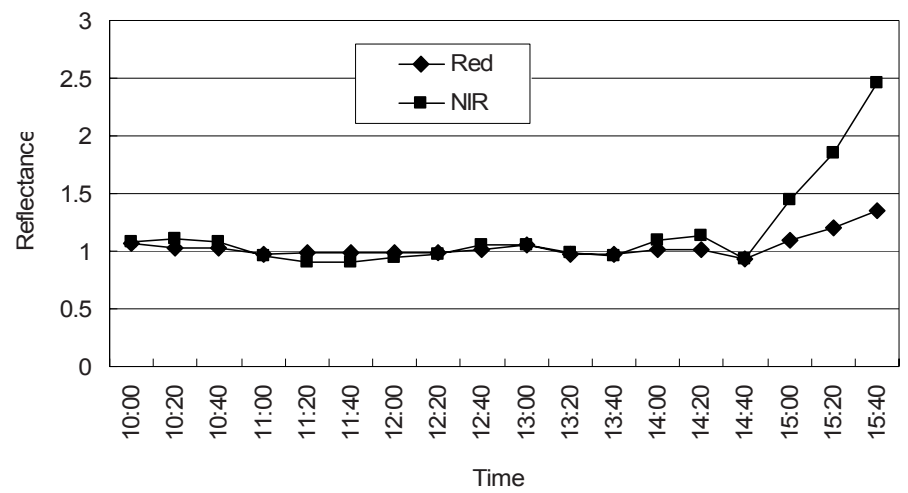

Figure 4. Measured reflectance of the white panel at different time of the day

As expected, the measured reflectance of the gray panel was found constantly lower than that of the white panel (Figure 5). However, a similar pattern to white panel result was found in this test that the measured reflectance data at NIR and red bands were around 0.35 between 10:00 AM and 14:40 PM, and significantly increased after15:00 PM. The standard deviation $(\sigma)$ of measured reflectance was 0.027 if excluding the last three measurements. Such result showed that the device satisfied the design requirement when the sunlight intensity was above a certain threshold value.

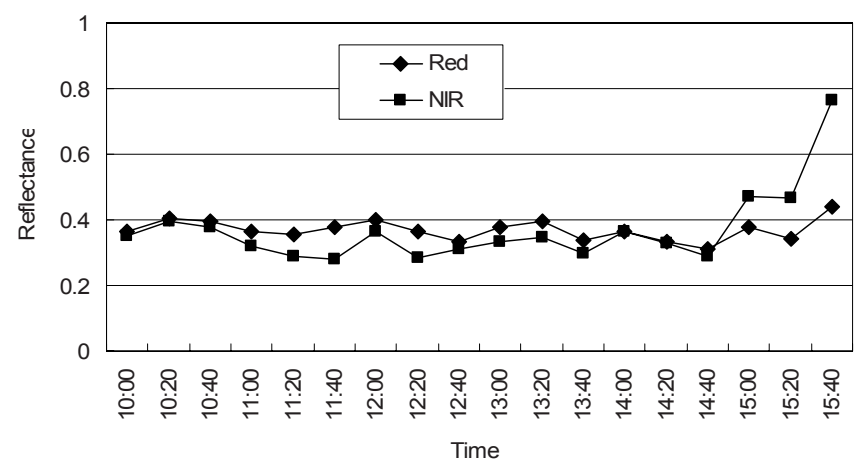

Figure 5. Measured reflectance of the gray panel at different time of the day 


\subsection{Field test results analysis}

A series of field tests were performed to evaluate performance of the developed device in a greenhouse environment. The instrument was placed about $10 \mathrm{~cm}$ above the crop plants. Total 24 samples were measured in this evaluation test. After measurement, leaf samples from the testing plants were collected for measuring the chlorophyll contents in a laboratory procedure. In this laboratory test, $0.4 \mathrm{~g}$ leaf sample was filled in test tubes with $25 \mathrm{ml}$ solution mixed acetone and ethanol in a 2:1 ratio. After place those tubes in dark room for 24 hours to dissolve the chlorophyll in the solution, the chlorophyll content of those processed samples were measured using an UVVIS Spectrophotometer.

The correlation coefficient between the NDVI readings from the developed device and the chlorophyll content measured by the UV-VIS Spectrophotometer is shown in Figure $6(r=0.81)$. The result shows that the NDVI readings from the developed device had a positive linear correlation with the chlorophyll content of tomato leaves, and the developed device could be used in practical measurement because of high correlation coefficient.

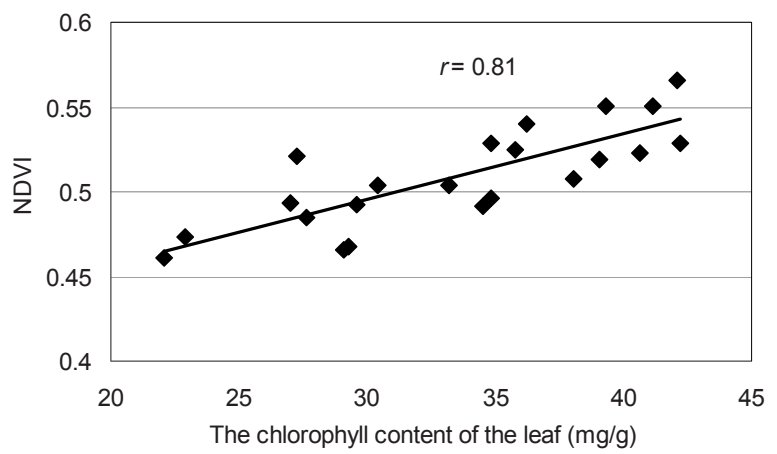

Figure 6. Correlation between the chlorophyll contents measured in laboratory procedure and NDVI readings from the developed device

\section{CONCLUSION}

A hand-held multispectral sensing device of crop chlorophyll content was developed. The initial test results indicated that the device could satisfied the design requirement $(\sigma<0.1)$ in field data collection. And the test results obtained in both laboratory and field environment showed that it could be 
used to detect the chlorophyll content level of tomato plants. Through calculating, the correlation coefficient between the NDVI readings from the developed device and the chlorophyll content data measured by the UV-VIS Spectrophotometer was $r=0.81$, which was high enough to be used in practical use. More intensive field tests is needed to verify and improve the developed device for its capability and limitation for actual field data collection.

\section{ACKNOWLEDGEMENTS}

Funding for this study was provided by National High Technology Research and Development Program of China (863 Program): Digital Agriculture (2007AA10Z207) and (2008AA10Z225).

\section{REFERENCES}

Campbell P, Middleton E M, McMurtrey J E, et al. Assessment of vegetation stress using reflectance or fluorescence measurements. Journal of Environmental Quality, 2007, 36(3): 832-845.

Cartelat A, Cerovic Z G, Goulas Y, et al. Optically assessed contents of leaf polyphenolics and chlorophyll as indicators of nitrogen deficiency in wheat (Triticum aestivum L.). Field Crops Research, 2005, 9(1): 35-49.

Heege H J, Thiessen E. On-the-go sensing for site-specific nitrogen top dressing. 2002 ASAE Paper No. 021113.

Kim Y, Reid J F, Hansen A, et al. Ambent Illumination effect on a spectral image sensor for detecting crop nitrogen stress. 2001 ASAE paper No. 01-1178.

Li M, Han D, Wang X. Spectral Analyzing technique and Applications. Science Press, 2006, Beijing, China.

Min M, Won S L, Yong H K, et al. Nondestructive detection of nitrogen in Chinese cabbage leaves using VIS-NIR spectroscopy. HortScience, 2006, 41(1): 162-166.

Noh H, Zhang Q, Han S, et al. Dynamic calibration and image segmentation methods for multispectral imaging crop nitrogen deficiency sensors. Trans. ASAE, 2005, 48(1): 393401.

Stone M L, Soile J B, Raun W R, et al. Use of spectral radiance for correcting in-season fertilizer nitrogen deficiencies in winter wheat. Trans. ASAE. 1996, 39(5): 1623-1631.

Sui R, Wilkerson J B, Hart W E, et al. Multi-spectral sensor for detection of nitrogen status in cotton. Applied Engineering in Agriculture, 2005, 21(2): 167-172.

Tucker C J. Red and photographic infrared linear combinations for monitoring vegetation. Remote Sensing of Environment, 1979, 8(2): 127-150.

Xue L, Cao W, Luo W, et al. Correlation between leaf nitrogen status and canopy spectral characteristics in wheat. Acta Phytoecologica Sinica, 2004, 28(2): 172-177. 